\title{
Propuesta de Actualización del RAOHE Incorporando Tecnologías de Fracturamiento Hidráulico, Reinyección de Recortes y Revisión de Límites Permisibles en la Gestión de Descargas Líquidas
}

\author{
Castillo Castro, Daniel Sebastián ${ }^{1,2, *(D)}$; Zambrano Carranza, Johnny Robinson ${ }^{1}$ iD ; Rivera Parra, José Luis ${ }^{1}$ \\ ${ }^{1}$ Escuela Politécnica Nacional, Departamento de Ingeniería en Petróleos, Quito, Ecuador \\ ${ }^{2}$ National Cheng Kung University, Laboratorio de Eficiencia Energética y de Recursos, Tainan, Taiwán
}

\begin{abstract}
Resumen: La legislación ambiental hidrocarburífera en el Ecuador inició en los 70 con el llamado pacto de caballeros y tuvo su momento cumbre con la expedición del RAOHE en 2001. En este punto, el RAOHE se consolidó como una herramienta para la explotación sustentable de los recursos hidrocarburíferos, y un garante de la estabilidad jurídica de una inversión extranjera que reactivase el sector petrolero. Sin embargo, 20 años después, con nuevos avances tecnológicos y garantías ambientales más restrictivas, se ha generado un desfase entre aquello que se encuentra regulado y la actual tecnología empleada en las operaciones de la industria. En este documento, se analizó esta discontinuidad normativa y de procesos desde el enfoque de la tecnología relacionada con el manejo y disposición de agua. Específicamente, en la revisión y análisis de límites permisibles en el manejo de descargas líquidas, reinyección de recortes y fracturamiento hidráulico. Este ensayo se apoyó en el estudio de fuentes de investigación primaria para la definición de sugerencias generales sobre la aplicación y gestión ante la entidad de gestión ambiental, a la espera de que esta información pueda aportar a la articulación de marcos técnicos más comprensibles.
\end{abstract}

Palabras clave: CRI, descargas líquidas, fracturamiento hidráulico, RAOHE, reinyección de recortes.

\section{RAOHE Review Proposal that Considers Technologies of Hydraulic Fracturing, Cuttings Reinjection and Revision of Permissible Limits in the Handling of Liquid Effluents}

\begin{abstract}
The Oil \& Gas environmental legislation in Ecuador has had a brief history. Its current regulation body is the RAOHE (Substitute Regulation to the Environmental Regulation for Hydrocarbon Operations in Ecuador) from 2001. At that time, the RAOHE was a robust mechanism that could guarantee the sustainable exploitation of hydrocarbon resources, while providing a solid ground for foreign capitals aiming to invest in the oil sector. Nonetheless, the RAOHE cannot be considered fit anymore to new technological advances and current international environmental regulations standards. Two decades without any update has generated a knowledge gap inside the RAOHE about what is regulated and what could be regulated under best management practices available in the industry. This paper aims to address this operational regulatory gap by performing an extensive literature review focused on wastewater management and better management practices. Specifically, this study evaluates the permissible limits in the handling and treatment of liquid discharges, cuttings reinjection and hydraulic fracturing. This paper provides a set of general suggestions and findings on the aforementioned areas. It is expected that this information can become a tool for policymakers to articulate more comprehensive technical regulatory frameworks.
\end{abstract}

Keywords: CRI, cuttings reinjection, hydraulic fracturing, liquid effluents, RAOHE.

\section{INTRODUCCIÓN}

Desde los años 70, muchos países desarrollaron un conjunto de normativas ambientales que apuntan a proteger el medioambiente de los efectos nocivos de la actividad humana. En la actualidad, estas mismas naciones aún son las pioneras en la formulación de regulaciones comprensibles y ejecutables que garantizan que los controles promulgados sean aplicados adecuadamente (Environmental Performance Index, 2018; WorleyParsons, 2014). Sin embargo, en Ecuador la realidad es muy distinta. A nivel cantonal (Gobiernos Autónomos Descentralizados), las ordenanzas pertinentes aún no disponen del nivel de madurez de otras instancias más avanzadas (Environmental Performance Index, 2018). El Reglamento Sustitutivo del Reglamento Ambiental para las Operaciones Hidrocarburíferas en el Ecuador (RAOHE) (2001) se sustentó 
sobre el artículo 86 de la Carta Magna de 1998 vigente hasta 2008 y marcó un referente en la regulación ambiental. El Reglamento se concibió bajo el ideal de constituirse como un mecanismo para la explotación racional de los recursos hidrocarburíferos y un garante de la estabilidad jurídica para la inversión extranjera; pero actualmente, el RAOHE se ha quedado muy por detrás de su objetivo principal: "superar las deficiencias del Reglamento Ambiental anterior" (Zehner, 2012). Transcurridas casi dos décadas desde su implementación, y sin ningún cambio sustancial evidente, existe un desfase entre lo que se encuentra regulado y la tecnología hoy empleada en las operaciones de la industria hidrocarburífera.

A partir de las oportunidades en I+D como mecanismo para la solución de problemas ambientales asociados a la industria del gas y petróleo, una importante proporción de esta discontinuidad corresponde a la tecnología relacionada con el manejo y disposición de agua de procesos. En este ámbito, en consideración del uso diversificado del agua durante las operaciones de Exploración y Producción (E\&P), parte de los desafíos al respecto incluyen comprender los impactos de su extracción desde los sistemas superficiales y subterráneos. Y consecuentemente, evaluar su rol activo durante la fase productiva de una operación de petróleo y gas no convencionales (U.S. Environmental Protection Agency, 2004). A su vez, este desfase regulatorio se afrontó desde un conjunto de aspectos que no se han actualizado en un periodo considerable de tiempo o que aún no han sido regulados. Estos incluyen: a) los límites permisibles en el manejo y tratamiento de descargas líquidas, b) la reinyección de recortes como método "cero-emisiones" de disposición de residuos, y c) el conjunto de tecnologías y técnicas de extracción de petróleo y gas no convencionales conocidas colectivamente como fracturamiento hidráulico.

El análisis se enfocó en estudiar las regulaciones que rigen la operatividad de las tecnologías de: a) fracturamiento hidráulico, y b) reinyección de recortes de perforación; con el propósito de definir sugerencias generales para la aplicación y gestión de estos procedimientos. Por otra parte, se analizaron los parámetros de control en la administración de descargas líquidas expuestos en la literatura científica y legislativa para generar una propuesta de actualización de los límites permisibles en el manejo de descargas líquidas considerados en el RAOHE. Finalmente, se espera que esta información aporte a la articulación de marcos técnicos, que sirvan de ancla para afianzar el empleo de prácticas ecológicas más eficientes; al mismo tiempo que permitan normalizar la existencia de ciertos vacíos jurídicos, explícitamente el abuso del derecho y la indefensión jurídica.

\section{FRACTURAMIENTO HIDRÁULICO}

En su artículo "The Frackers and the Birth of an Energy Boom”, Bryan Burrough (2013) menciona "uno podría argumentar que, exceptuando el internet, el fracturamiento hidráulico ha sido el avance tecnológico más importante en las últimas dos décadas". Y una vez que se lo piensa, puede tener razón.
En medida de su relevancia, los esfuerzos por regular el fracturamiento hidráulico esencialmente han sido direccionados a normar una actividad mediáticamente descrita como agresora potencial de los cuerpos de agua (superficiales y subterráneos) y de la geología de sitios hidráulicamente fracturados (U.S. Environmental Protection Agency, 2004). Regularmente, la desavenencia sobre el fracturamiento hidráulico surge a partir de un desajuste en su concepto, una brecha en el lenguaje. En términos de ingeniería, el fracking conceptualiza una actividad específica de estimulación, limitada a la generación de grietas en la roca a partir de la inyección de fluido; mientras que para muchos grupos ambientalistas y ciudadanos preocupados, ha llegado a representar casi todas las fases del ciclo de desarrollo de un pozo. A fin de dilucidar los malentendidos sobre el tema, en esta sección se expone la dinámica del proceso de estimulación, consideraciones ambientales concomitantes y un sumario de los principales estándares manejados dentro de las operaciones de fracturamiento hidráulico (Alberta Energy Regulator, 2018; U.S. Geological Survey, 2014).

\subsection{Estudio de Normativas Vigentes}

El fracturamiento hidráulico consiste en el bombeo de un fluido de fracturación, compuesto por agua, aditivos y partículas apuntalantes, a una formación a una velocidad y presión calculadas y predeterminadas para generar fracturas o grietas en el estrato objetivo. El trabajo conjunto de estos compuestos permite que la fractura se abra paso dentro de la roca (Canadian Association of Petroleum Producers, 2017; Viswanathan et al., 2015). La primera etapa del fracturamiento hidráulico inicia con su diseño, el cual implica identificar las propiedades de la formación objetivo, incluida la presión de fractura y la longitud deseada de las fracturas (King, 2012). Una vez obtenido el modelo del fracturamiento, se continua con la evaluación del pozo, equipo del pozo y equipo de fracturamiento hidráulico a las condiciones de presión y flujo a las cuales estarán sujetos durante la operación (ALL Consulting \& Ground Water Protection Council, 2009). Las pruebas incluyen la evaluación del casing y cemento del pozo durante la perforación y completación, y la ejecución de pruebas de presión al equipo de fractura hidráulica (Harper, 2007).

Posteriormente, al llevar la operación al campo es importante notar que el fracturamiento hidráulico puede ejecutarse tanto en pozos horizontales como verticales. La diferencia residirá en las etapas del proceso, es decir, cada etapa de un tratamiento de fractura de un pozo horizontal es equivalente a un tratamiento de fractura para un pozo vertical.

El procedimiento estándar establece que en un inicio debe ejecutarse un procedimiento de "mini frac" - una prueba diagnóstica de inyección-admisión- seguida del bombeo secuencial de un tratamiento ácido, un bache de agua de baja fricción -slick water, que facilita el flujo del apuntalante-y varias sub-etapas de apuntalante y agua. La configuración de estas sub-etapas destaca puesto que se inicia con el bombeo de un gran volumen de agua con arena de malla fina, y continua con el decremento gradual del volumen de agua y el aumento paulatino del tamaño de grano del apuntalante (Palisch et al., 2010). Después de la finalización de la sub-etapa final de 
apuntalante grueso, el pozo y el equipo se lavan con la inyección de un volumen de agua dulce lo suficientemente grande para eliminar el exceso de apuntalante del equipo y del pozo.

Los fluidos de fractura consisten principalmente en agua y arena (alrededor del 98\%), pero también incluyen una variedad de aditivos. La mayoría de estos aditivos cuentan con usos exclusivamente industriales, presentando riesgos para la salud sólo cuando son manipulados imprudentemente, al omitir los procedimientos de seguridad correspondientes (The Royal Society \& Royal Academy of Engineering, 2012). Un tratamiento típico emplea concentraciones muy bajas de entre 3 y 12 aditivos dependiendo de las características del agua y la formación que se fracture. La perforación y la fracturación hidráulica de un pozo puede requerir típicamente de 2 a 4 millones de galones de agua (Gallegos et al., 2015; Satterfield et al., 2008). Sin embargo, aun cuando estos volúmenes de agua necesarios para perforar y estimular los pozos de gas de esquisto parecen inusualmente grandes, generalmente representan un pequeño porcentaje del uso total de agua en las cuencas correspondientes. No obstante, al requerirse en un período relativamente corto, su extracción puede afectar a la fauna marina, suministros de agua municipales y otras industrias (Kondash \& Vengosh, 2015). Para ello, en consideración de las condiciones complejas en la obtención de agua, los estados y operadoras han ideado medidas compensatorias tales como la diferenciación de flujos estacionales y planes locales de administración del recurso hídrico (identificación de suministros de agua abundantes) (Weston, 2008).

La contaminación de los recursos hídricos es uno de los aspectos más preocupantes para la opinión pública. Esta podría darse debido a los químicos adicionados al fluido de fracturamiento hidráulico, tanto como a derrames, fugas o migración del fluido de fractura o agua de formación (Vengosh et al., 2014). A la fecha, dentro de la literatura científica aún no se ha reportado ninguna evidencia definitiva sobre la contaminación de acuíferos debido a la migración de los fluidos inyectados a los reservorios a lo largo de las fracturas creadas por el fracturamiento hidráulico (Gallegos et al., 2015; Mauter et al., 2014). Sin embargo, al haberse encontrado evidencia sobre la migración de gas a lo largo de pozos construidos precariamente (Vengosh et al., 2014), se infiere la posibilidad de la migración de otros fluidos por medio de los mismos mecanismos. Al mismo tiempo, también es cuestionado el impacto que tiene la inyección de grandes volúmenes de agua sobre la integridad del pozo (Vengosh et al., 2014).

Asimismo, en la industria existe incertidumbre alrededor de ciertos incidentes sísmicos que pueden estar asociados con la disposición del agua de producción extraída. Es propicio señalar que, previamente, la sismicidad inducida también ha sido vinculada a una serie de otras actividades humanas (proyectos geotérmicos, extracción minera y construcción). En

\footnotetext{
${ }^{2}$ La política basada en los estándares API incluye entre los principales lineamientos: i) Revisión mandatoria de los diseños del pozo, i.e. inspecciones in situ, según corresponda, para garantizar que los pozos se construyan de acuerdo con el diseño acordado; ii) Empleo de técnicas eficaces, e.g. método de bombeo y sello, en donde el cemento debe alcanzar una resistencia a la
}

ese contexto, la sismicidad inducida puede ocurrir cuando existe una falla geológica bajo estrés. El aumento de la presión mientras se inyecta el fluido (agua de producción) puede liberar la falla y esto permitir el deslizamiento. Lo que a su vez resulta en ciertas liberaciones de energía muy similares a aquellas generadas por los terremotos, pero que debido a diferencias significativas en su frecuencia y magnitud, son catalogadas como eventos cizallamiento mas no pequeños sismos (Frohlich et al., 2010).

Por último, resulta importante considerar que debido a que el fracturamiento hidráulico no es una operación de procedimiento universal, las suposiciones y generalizaciones concernientes al uso del agua dentro de las operaciones de fracturamiento hidráulico y el potencial de los impactos ambientales deben tomarse con precaución.

\subsection{Recomendaciones}

A partir del trabajo conjunto entre industria, agencias reguladoras y público, se ha desarrollado una serie de documentos que abordan los problemas de gestión de riesgos que acompañan a la construcción y gestión de pozos no convencionales (e.g. "Shale Gas Extraction in the UK: a review of hydraulic fracturing”, "API's Guidance Documents on Hydraulic Fracturing Operations", "An Investor Guide to Disclosing Risks from Hydraulic Fracturing Operations", etc...). Estas directrices proveen una fuente fiable de soluciones ambientales innovadoras ante los nuevos desafíos de la revolución tecnológica del fracking. A partir de su estudio y análisis, se presentó una propuesta técnica razonable a la regulación del fracturamiento hidráulico que propende a garantizar el desarrollo sostenible de la actividad industrial. El ámbito de la propuesta se encuentra direccionado a siete conjuntos de prácticas recomendadas, listando entre ellas: a) el aseguramiento de la integridad del pozo; b) la detección de contaminantes en el agua subterránea; c) la detección de fugas potenciales de gas; d) la mitigación de la sismicidad inducida; e) la gestión integral del agua y de los fluidos de fractura y de retorno; y, f) el manejo de riesgos ambientales.

La consecución del primer punto busca anular los incidentes por fugas accidentales de gases y fluidos peligrosos desde las locaciones. Su mecanismo principal es la implementación de una política estándar ${ }^{2}$ sobre la construcción integral de pozos para eliminar virtualmente los riesgos de fugas de metano y químicos que surgen de la construcción deficiente (American Petroleum Institute, 2009).

Para el segundo y tercer objetivo, por una parte, se requiere identificar las condiciones de referencia en los cuerpos de agua vecinos y las fuentes para agua potable. Y por otra, monitorear de manera rutinaria su calidad durante la fracturación y la producción, incluyendo riesgos potenciales de metano biogénico cercano a la superficie (Canadian Association of Petroleum Producers, 2012a; Kiparsky \& Foley, 2013). Se recomienda que los estudios sean conducidos por laboratorios

compresión de 300 psi en 24 horas y 800 psi en 72 horas; iii) Asentamiento del revestidor superficial a una profundidad no menor a 150 pies por debajo del acuífero aprovechable más profundo.; y, iv) Testeo rutinario de pozos pre y post fractura (registros de cemento) (American Petroleum Institute, 2009). 
independientes para garantizar la transparencia del reporte (ALL Consulting \& Ground Water Protection Council, 2009).

Para el logro del cuarto punto, se establece que es deber del servicio geológico -o entidad análoga- realizar evaluaciones en el territorio nacional. Esto, a fin de caracterizar estreses, identificar fracturas en las formaciones de interés y establecer una base de datos nacional donde se identifique las zonas más apropiadas para las locaciones de pozos hidráulicamente fracturados (Rutqvist et al., 2007).

El quinto objetivo está parcialmente orientado a extraer el mínimo de agua necesaria para realizar operaciones de fracturamiento. Sus estrategias incluyen sacar ventaja de las diferencias estacionales en los cauces naturales (ALL Consulting \& Ground Water Protection Council, 2009), emplear fuentes no potables (e.g. acuíferos salinos, aguas residuales tratadas o aguas de reflujo) (American Petroleum Institute, 2010; Kiparsky \& Foley, 2013) e innovar en la utilización de fluidos de fractura no acuosos (e.g. gas licuado de petróleo gelificado, dióxido de carbono y espumas nitrogenadas) (The Royal Society \& Royal Academy of Engineering, 2012).

Además, abarca la evaluación y gestión de aditivos en los fluidos de fractura y la divulgación de aditivos en los fluidos de fractura. Los requerimientos clave incluyen la eliminación casi absoluta de los productos químicos tóxicos utilizados en las operaciones de fracturamiento y la divulgación exhaustiva de los objetivos de reducción (Barrios, 2012; Kiparsky \& Foley, 2013; Liroff et al., 2017). También, señala que las compañías se encuentran en la obligación de revelar públicamente, en bases individuales pozo por pozo, el nombre común y el número único de identificación química (CAS) de cada ingrediente químico de los fluidos de fracturación utilizados o planificados para uso en sus pozos (Canadian Association of Petroleum Producers, 2012b).

En lo referente al transporte, manipulación, almacenamiento y disposición de fluido de fractura, agua de producción, flujo de retorno y desecho del fluido de fractura, se recomienda el empleo de tanques de acero o sistemas de circuito cerrado siempre que sea posible. Con ello se mitiga el riesgo de contaminación (evitando el empleo de zanjas y piscinas de retención). Otro factor clave en esta materia incluye la evaluación de los flujos de retorno y agua producida para garantizar un tratamiento o eliminación seguros (American Petroleum Institute, 2009; Canadian Association of Petroleum Producers, 2012c).

Para el logro del sexto punto, los requerimientos resaltan lo señalado por el capítulo IV "Estudios Ambientales" del RAOHE. Indicando que, los estudios de impacto ambiental (EIA) deben ser mandatorios para todas las operaciones de fracturamiento hidráulico, considerando una participación activa por parte de la comunidad local. El EIA debe evaluar los riesgos a lo largo de todo el ciclo de exploración y producción, incluyendo la disposición de desechos, abandono de pozos y riesgos sísmicos (ALL Consulting \& Ground Water Protection Council, 2009; American Petroleum Institute, 2013,
2014; The Royal Society \& Royal Academy of Engineering, 2012).

De momento, aun cuando en Ecuador sólo se han realizado operaciones de fracturamiento en yacimientos convencionales, la existencia de reservas hidrocarburíferas no convencionales no se lo descarta (basados en las prospecciones de Cuervos y Cerrajón) (Andagoya \& Pérez, 2013). Y fundamentados en las similitudes entre ambas aplicaciones del fracking, esta circunstancia no hace más que brindar más trascendencia a este estudio sin llegar a invalidar las recomendaciones aquí propuestas.

La discusión circundante al fracturamiento hidráulico toma mayor relevancia al sopesar los beneficios económicos frente a los posibles impactos en las personas y el medio ambiente. No existe duda que este juega un papel vital en el suministro de energía contemporáneo. Empero, los riesgos de salud, seguridad y ambiente asociados al fracturamiento hidráulico como un medio de extracción de recursos no convencionales pueden llegar a ser manejados efectivamente en Ecuador siempre que las mejores prácticas operacionales sean implementadas y aplicadas a través de la regulación apropiada. Ninguna de las propuestas de prácticas recomendadas es tan novedosa como para ser descartada de aplicarse por operadores prudentes (Mordick, 2012). El camino está a medio trazar, y es posible para las compañías ir más allá en términos de mejores prácticas a fin de mantener su credibilidad y deber con la preocupación pública.

\section{REINYECCIÓN DE RECORTES}

La gestión general de residuos está subordinada a la jerarquía de minimización, reducción, reciclaje y recuperación, tratamiento y su disposición o descarga final (Abou-Sayed \& Guo, 2001). Así cuando en 1993 en Noruega se incrementaron obligatoriamente las regulaciones para la disposición de recortes de perforación en áreas marinas, la inyección de recortes de perforación (CRI o DCI), i.e., la disposición de los desechos asociados a la perforación dentro de una formación receptora hidráulicamente fracturada surgió como una tecnología emergente ambiental y económicamente fiable (Guo et al., 2005).

Su campo de aplicaciones es tan amplio que en la actualidad en Ecuador esta práctica ya se ha llevado a cabo exitosamente en contadas ocasiones, y tal ha sido su eficiencia, que ha permitido poner sobre la mesa la articulación de marcos técnicos que avalen estas operaciones (Romero et al., 2016). En este contexto, a continuación, se establece un sumario de las prácticas más relevantes recomendadas en el aseguramiento de CRI, por medio de una revisión detallada de los procedimientos operacionales, casos de estudios y reformas regulatorias internacionales.

\subsection{Estudio de Normativas Vigentes}

En un modo pragmático, todas las operaciones de inyección de recortes son procesos por baches, en los cuales se preparan periódicamente volúmenes moderados ${ }^{3}$ de lechada, i.e.

\footnotetext{
2002).

Aproximadamente 200 BPD inyectados en tasas de 1 a 6 BPM (Keck,
} 
recortes triturados en presencia de agua, los cuales son inyectados con un determinado tiempo de separación o cierre entre cada inyección. (Guo \& Abou-Sayed, 2003) Figurativamente, después de la primera inyección se crea una fractura planar dentro de la formación receptora, sobre la cual la lechada filtra al mismo tiempo que esta (la fractura) se cierra, atrapando los recortes y lodo dentro de la grieta generada. Con las inyecciones consiguientes se abren más fracturas derivadas de la fractura original. Estas conforman una red de fracturas interconectadas conceptualizadas como el dominio de eliminación (Moschovidis et al., 1994).

La reinyección de recortes se desarrolla bajo dos esquemas estándar, inyección anular e inyección en un pozo dedicado (completación de tubería de producción y empacador). La inyección anular es más común en locaciones remotas o ecológicamente sensibles, donde los recortes se inyectan en el anular del pozo que está siendo perforado. Del otro lado, el esquema de un pozo dedicado suele ser más típico de las operaciones de inyección permanente a largo plazo. Frecuentemente, estos pozos están en servicio durante varios años (Keck, 2002).

En la actualidad, en su mayoría, los programas de reinyección se desarrollan sin presentar contratiempos serios. Sin embargo, hay ocasiones en las que las operaciones de CRI se ven comprometidas por numerosos factores. Algunos casos incluyen el bloqueo de la trayectoria existente hasta el estrato de disposición por debajo de la tubería de revestimiento o del espacio anular. Asimismo, aunque su ocurrencia es rara y se limita a operaciones offshore, existen otros casos en los que las lechadas de inyección migran a través de fracturas naturales, fracturas inducidas hidráulicamente o secciones del pozo cementadas en forma deficiente, volviendo al lecho marino (Guo et al., 2005; Nagel \& Strachan, 1998).

Análogamente, a fin evitar estas circunstancias existe un espectro metodológico aún más extenso que garantiza el éxito de una operación de CRI. El mismo que incluye la planificación cuidadosa del trabajo, diseño e ingeniería adecuados, monitoreo continuo, gestión de riesgos y procedimientos operacionales de contingencia (Guo et al., 2007). En primer lugar, el estudio de factibilidad identifica los posibles riesgos operacionales y propone las medidas de mitigación correspondientes. Sus requerimientos mínimos incluyen la descripción de la geología subsuperficial, caracterización de los recortes (tasa de generación, volumen total, propiedades químicas y físicas), detalle de las facilidades requeridas para la operación y, análisis de riesgos, opciones de mitigación y procedimientos operacionales.

En segunda instancia, el diseño y simulación de la operación permiten determinar la reología de la lechada, comportamiento de los estreses de formación y capacidad de almacenamiento. Finalmente, una vez que el diseño de la operación está en su lugar, es necesario monitorear las operaciones activamente. El control de calidad y los ajustes de propiedades reológicas y

\footnotetext{
${ }^{4}$ Las mejores prácticas de cementación integran la buena centralización de los revestidores, colocación óptima, eliminación eficaz del lodo y espaciadores, definición apropiada de los topes de cemento para un aislamiento efectivo y rotación de la tubería durante la cementación (AbouSayed \& Guo, 2002).
}

físicas específicas del sitio son cruciales para mantener la integridad de la operación y completar con éxito el proyecto (Abou-Sayed \& Guo, 2002; Saasen et al., 2001). Los parámetros que deben considerarse dentro del monitoreo incluyen, el registro continuo de las propiedades de la lechada inyectada (composición, concentración, densidad, etc...), de la presión de fondo y durante la inyección y en los periodos prolongados de cierre y el análisis del comportamiento de la formación y la fractura durante la inyección y cierre (Bruno et al., 2000).

\subsection{Recomendaciones}

El aseguramiento de una operación de reinyección de recortes es un objetivo complejo de alcanzar. No obstante, distintas experiencias operativas han demostrado que el seguimiento de un proceso bien planificado e integrado, dentro del que se consideren barreras y controles para evitar que los riesgos se propaguen y causen consecuencias inestimadas, contribuye de manera efectiva en la acometida de este tipo de operaciones (Abou-Sayed \& Guo, 2002; Guo \& Abou-Sayed, 2003). Una recopilación de las mejores estrategias para el aseguramiento de una operación de CRI, incluye: a) la contención de fluidos, b) el diseño del procedimiento operacional, y c) el monitoreo post-implementación. Estas recomendaciones proporcionan un mecanismo para afianzar la seguridad industrial y ambiental del procedimiento, en tanto que permanecen flexibles para su aplicación en distintos escenarios.

En primera instancia, la irrupción de la lechada en superficie y en acuíferos someros es el principal riesgo ambiental que plantea la CRI (Abou-Sayed \& Guo, 2002). Al respecto, la mayor medida de mitigación consiste diseñar y modelar la operación de manera precisa para obtener una comprensión de las características principales que afectarán el crecimiento de la fractura y consideraciones asociadas a largo plazo (Guo \& Abou-Sayed, 2003; Guo et al., 2007).

Como prácticas recomendadas para la selección de la formación de disposición se incluyen: a) la selección un estrato con estreses bajos (arcillas y lutitas) limitado por encima y por debajo de zonas con altos estreses (areniscas) y alejado de fallas o sus prospectos; $y, b$ ) la verificación de la integridad del pozo de inyección. Localmente, como resultado del estudio llevado en el campo Apaika, (Hernandez et al., 2015) concluyeron que las formaciones más adecuadas para albergar un proyecto de CRI son Tiyuyacu Superior, T superior e inferior, U superior e inferior y Hollín.

Con respecto a la integridad del pozo, la preferencia general es seleccionar pozos que provean un acceso adecuado a la formación de interés; con una buena calidad de cemento a lo largo de toda la zona de eliminación ${ }^{4}$; y una fecha de perforación lo más reciente posible para minimizar la reacción de la formación y deterioro de la inyectividad con el tiempo ${ }^{5}$ (Gumarov et al., 2014). Adicionalmente, se señala que el diámetro efectivo seleccionado debe proveer una velocidad y

\footnotetext{
${ }^{5} \mathrm{O}$ a su vez, un pozo en el cual se haya dejado el espacio anular con una salmuera libre de sólidos que sea compatible con la formación, a fin de evitar la dispersión química y la potencial obstrucción de los poros (Abou-Sayed \& Guo, 2001)
} 
régimen de flujo óptimos dentro del pozo, minimizando la precipitación de sólidos.

Por otro lado, en correspondencia al diseño operacional, las consideraciones más importantes son el diseño de la reología de la lechada y el procedimiento operacional. Como regla general, la tasa de inyección debe ser igual o ligeramente mayor que la tasa de generación de recortes. Esto minimizará la necesidad de tanques de almacenamiento. Consideraciones adicionales incluyen: desplazar todo el volumen del anular o tubería de inyección después de cada bache para evitar el asentamiento de partículas y el taponamiento de la zona de eliminación. Adicionalmente, se recomienda diseñar el equipo de completación y superficie reconociendo su interacción directa con el sistema de inyección. El estándar incluye un cabezal y tuberías que se adapten a las necesidades de la operación, con materiales anticorrosivos superpuestos a las áreas de sellado y juntas extra gruesas a modo de evitar riesgos de falla del equipo ocasionados por el desgaste erosivo (Guo et al., 2007).

Las últimas recomendaciones corresponden a medidas de mitigación tales como el monitoreo y verificación de operaciones. Su estándar más valorado es el empleo del análisis de datos operativos (propiedades de la lechada, resultados de las pruebas de sedimentación y presión de inyección) para validar los resultados del modelo preparado durante la evaluación de factibilidad y simulación. De esta manera es posible la generación de alertas tempranas en miras a mejorar los procedimientos y parámetros operativos para evitar consecuencias no deseadas (Comisión OSPAR, 2001; Guo et al., 2007).

Las soluciones actuales sobre el manejo de desechos sólidos distan de la perfección, pero son mucho mejores que las disponibles hace algunas décadas. Por ello, es fundamental que las operaciones sigan las mejores prácticas de evaluación, diseño, implementación y monitoreo. Esto es particularmente importante para los proyectos de CRI en áreas remotas o ambientalmente sensibles ya que a menudo esta tecnología es la única opción económica y cualquier percance con la operación podría resultar muy costosa técnica, ambiental y socialmente.

\section{REVISIÓN LÍMITES PERMISIBLES EN EL MANEJO DE DESCARGAS LÍQUIDAS}

Uno de los desafíos del siglo XXI ha sido satisfacer la demanda energética mundial de una manera segura $\mathrm{y}$ sustentable. Para ello, las compañías de petróleo y gas y los organismos gubernamentales han implementado regulaciones en miras de mejorar la sostenibilidad de la industria (Doric \& Dimovski, 2018). Así, debido a la creciente demanda del recurso hídrico, la regulación sobre el manejo y tratamiento de aguas es uno de los ejes en este ámbito (Grant et al., 2012). Sin embargo, cuando el conjunto de herramientas y directrices ambientales regulatorias son muy complejas, esto lleva a incoherencia entre políticas e incertidumbre para las empresas.

Localmente, cuando se propuso el Reglamento Ambiental para las Operaciones Hidrocarburíferas en el Ecuador (RAOHE), éste buscó superar las deficiencias de su antecesor, a través de revisiones y adaptaciones en la metodología de trabajo. En este ámbito, uno de los aspectos más impactantes fue el cambio radical respecto al listado de parámetros para el monitoreo y control de descargas. Previamente, el número de parámetros de monitoreo correspondiente a aguas de producción era exorbitante en comparación de otras legislaciones, ocasionando que las rutinas de trabajos se volviesen poco operacionales (Zehner, 2012).

El RAOHE vigente condensa toda la materia de límites permisibles en el Capítulo XII, artículo 86, y dicta:

"Los sujetos de control y sus operadoras y afines en la ejecución de sus operaciones, para descargas líquidas [...], cumplirán con los límites permisibles que constan en los Anexos No. 1, 2 y 3 de este Reglamento, los cuales constituyen el programa mínimo para el monitoreo ambiental interno ...".

Más allá, es primordial reconocer que las descargas provenientes de los sistemas de aguas residuales contienen contaminantes extensivamente persistentes, así como la existencia de problemas ambientales y salubres directamente derivados del tratamiento ineficiente de las mismas (Government of Canada, 2015). Sobre la base de este hecho, se propone la actualización de los límites permisibles y consideración de lineamientos sobre la administración de efluentes líquidos. Propuesta que tiene como objetivo la limitación de los efectos potencialmente dañinos que estas sustancias puedan tener sobre los ecosistemas y la salud humana.

\subsection{Estudio de Normativas Vigentes}

La metodología de trabajo empleada desarrolló un marco comparativo en base a fuentes bibliográficas secundarias y extrajo información de fuentes primarias como respaldo a aquella encontrada en primera instancia. En razón a esta mecánica, mediante el empleo de los indicadores EPI (Environmental Performance Index, 2018) y CAPP (WorleyParsons, 2014) fue posible recopilar la gran mayoría de aspectos relevantes respecto al manejo de descargas líquidas. Las metodologías empleadas por EPI y CAPP permiten analizar el desempeño (de la política ambiental) en diferentes niveles ecológicos y sociales y escalar razonablemente las políticas ambientales marco sobre la base de la realidad industrial correspondiente a cada nación. En la siguiente sección, la Tabla 1. expone los lineamientos estipulados en el RAOHE (Ministerio de Energía y Minas, 2001) frente a aquellos de otras naciones seleccionadas como sujetos de referencia, entre las que se incluye Australia (Department of Environment and Heritage Protection, 2014a, 2014b), Canadá (Alberta y Ontario) (Environment and Sustainable Resource Development, 2014; Minister of Fisheries and Oceans, 2002), Estados Unidos (Luisiana) (Louisiana Department of Environmental Quality, 2017) y Colombia (Ministerio de Ambiente y Desarrollo Sostenible, 2015). Entre los justificativos pertinentes se encuentran los niveles de actividad en el sector de gas y petróleo de estas regiones, y la madurez y apertura comparativa que cada uno de estos gobiernos presenta. 
Cabe acotar de manera enfática que los lineamientos de la Tabla 1 son generales, puesto que dentro del entorno natural existe una variabilidad inherente, la cual induce al empleo de información específica para el desarrollo de pautas e índices apropiados de calidad ambiental en determinadas ocasiones. Este razonamiento puede ser ampliamente sustentado bajo el amparo de la ecotoxicología, un estudio sobre cómo los químicos (usualmente de origen humano o antropogénico) se comportan dentro del medio ambiente y cómo afectan a los organismos que allí se desarrollan.

\subsection{Recomendaciones}

En la industria hidrocarburífera, existen sustancias que ocurren de forma natural o que se evidencian a través de procesos naturales, como el cadmio, el mercurio y los hidrocarburos aromáticos policíclicos (HAP), cuya eliminación completa de emisiones, descargas y fugas de todas las fuentes potenciales resulta imposible (Burgess, 2013). Las concentraciones de estos químicos tóxicos y nutrientes biológicamente disponibles en exceso pueden derivar en diversos problemas como floraciones de algas tóxicas, pérdida de oxígeno, muerte de peces, pérdida de biodiversidad y pérdida de lechos de plantas acuáticas (Voutsa et al., 2001). Así, al momento de elaborar las directivas individuales pertinentes, el entendimiento apropiado de la toxicología de los contaminantes ambientales debe ser adecuadamente considerado y las medidas deben tener como objetivo el cese de las emisiones, vertidos y pérdidas en el agua de las sustancias peligrosas prioritarias que se derivan de las actividades humanas (Burgess, 2013).

Además, la naturaleza de estos elementos tóxicos también puede ser antropogénica o biogénica. Dentro de la literatura existen muchas aproximaciones respecto al manejo y control de emisiones, mecanismos de atenuación, y herramientas de remediación. A continuación, se compilan criterios valiosos (pertinentes, relevantes y actuales) para establecer lineamientos idóneos para sostener la vida en un medio genérico.

- Un pH dentro de un rango de 6.5 a 9.0 es el más apropiado para el sostenimiento de comunidades de peces (Sparling, 2016a; U.S. Environmental Protection Agency, 2018).

- Jouanneau et al. (2014) y Safari et al., (2013) coinciden en que concentraciones menores a $60 \mathrm{mg} / \mathrm{L}$ en la descarga de efluentes a cuerpos de agua dulce pueden ser tomadas como buenas señales de cumplimiento ambiental. Adicionalmente, se anota que bajas concentraciones de oxígeno disuelto, cuando combinado con la presencia de sustancias tóxicas pueden derivar en estrés de los ecosistemas acuáticos (puesto que la concentración de ciertos elementos se incrementa por bajas concentraciones de oxígeno disuelto). Asimismo, que las altas temperaturas del agua también incrementan los efectos adversos en la biota asociada con bajas concentraciones de oxígeno disuelto (Alabaster, 1982b, 1982a).

- La "Directiva de Nitratos"(Jarvie et al., 1998), señala que un cuerpo superficial de agua fresca puede ser clasificado como no contaminado cuando la concentración de amoniaco (como nitrógeno total) no excede los $11.3 \mathrm{mg} / \mathrm{L}$.

- La Comisión Oslo - París (OSPAR) establece un objetivo límite de descarga de $30 \mathrm{mg} / \mathrm{L}$ de hidrocarburos totales en el agua de producción (Commision OSPAR, 2014).

Tabla 1. Matriz comparativa del RAOHE frente a varias regulaciones ambientales internacionales

\begin{tabular}{|c|c|c|c|c|c|c|c|c|}
\hline \multirow[b]{2}{*}{ PARÁMETRO } & \multirow[b]{2}{*}{ UNIDADES } & \multicolumn{2}{|c|}{ ECUADOR } & \multicolumn{2}{|c|}{ COLOMBIA } & \multirow{2}{*}{$\begin{array}{c}\text { ESTADOS } \\
\text { UNIDOS } \\
\text { (LOUISIANA) }\end{array}$} & \multirow[b]{2}{*}{ CANADÁ } & \multirow[b]{2}{*}{ AUSTRALIA } \\
\hline & & $\begin{array}{l}\text { VALOR LÍMITE } \\
\text { PERMISIBLE }\end{array}$ & PROMEDIO ANUAL & EXPLORACIÓN & PRODUCCIÓN & & & \\
\hline $\mathrm{pH}$ & Un. de $\mathrm{pH}$ & $5,0-9,0$ & $5,0-9,0$ & $6,0-9,0$ & $6,0-9,0$ & $6,0-9,0$ & $6,5-9,0$ & $6,0-8,0$ \\
\hline DQO & $\mathrm{mg} / \mathrm{L}$ & $<120,00$ & $<80,00$ & $<200,00$ & $<60,00$ & $<125,00$ & $<25,00^{*}$ & $<120,00$ \\
\hline Sólidos totales (ST) & $\mathrm{mg} / \mathrm{L}$ & $<1700,00$ & $<1500,00$ & $<1500,00$ & $<1500,00$ & & Descriptivo $^{d *}$ & \\
\hline Fenoles & $\mathrm{mg} / \mathrm{L}$ & $<0,15$ & $<0,10$ & $<0,20$ & $<0,20$ & & $<0,004$ & \\
\hline Conductividad Eléctrica & $\mu \mathrm{S} / \mathrm{cm}$ & $<2500,00$ & $<2000,00$ & & & & & $<106,00$ \\
\hline HTP & $\mathrm{mg} / \mathrm{L}$ & $<20,00$ & $<15,00$ & $<10,00$ & $<10,00$ & $<15,00$ & $<30,00$ & \\
\hline Nitrógeno Total (N) & $\mathrm{mg} / \mathrm{L}$ & $<20,00$ & $<15,00$ & $<10,00$ & $<10,00$ & & Ecuación ${ }^{e}$ & $<0,668$ \\
\hline Bario (Ba) & $\mathrm{mg} / \mathrm{L}$ & $<5,00$ & $<3,00$ & & & & & \\
\hline Cromo (Cr) & $\mathrm{mg} / \mathrm{L}$ & $<5,00$ & $<0,40$ & $<0,50$ & $<0,50$ & $<0,50$ & $<0,10$ & $<0,10$ \\
\hline Plomo (Pb) & $\mathrm{mg} / \mathrm{L}$ & $<0,50$ & $<0,40$ & $<0,20$ & $<0,20$ & & & $<0,20$ \\
\hline Vanadio (V) & $\mathrm{mg} / \mathrm{L}$ & $<1,00$ & $<0,80$ & $<1,00$ & $<1,00$ & & & $<0,10$ \\
\hline HAPs & $\mathrm{mg} / \mathrm{L}$ & $<0,0003^{*}$ & $<0,0002^{*}$ & $<0,01$ & $<0,01$ & & & \\
\hline BTEX & $\mathrm{mg} / \mathrm{L}$ & & & $<0,01^{\mathrm{c}}$ & $<0,01^{\mathrm{c}}$ & $<4,8675$ & $<0,20^{*}$ & \\
\hline Turbidez & NTU & & & & & & Descriptivo $^{f}$ & $2,0-200,0$ \\
\hline Temperatura & ${ }^{\circ} \mathrm{C}$ & Condici & atural $\pm 3^{*}$ & & & & Descriptivo ${ }^{g}$ & \\
\hline Color & $\mathrm{CU}$ & Inapreciable & solución $1: 20^{*}$ & & & & Descriptivo $^{\mathrm{h}}$ & \\
\hline
\end{tabular}

Notas:

${ }^{a}$ En cualquier momento

${ }^{b}$ Promedio de las determinaciones realizadas en un año conforme a la frecuencia de monitoreo establecida en el Art. 11 del RAOHE

'En los casos en que el vertimiento puntual de aguas residuales se realice en un cuerpo de agua superficial receptor o en un tramo de este, que tenga como destinación el uso del agua para consumo humano y doméstico, y pecuario la concentración de Hidrocarburos Aromáticos Policíclicos (HAP) en el vertimiento puntual de aguas residuales deberá ser menor o igual a 0,01 mg/L para aquellas actividades que lo tienen definido como de análisis y reporte.

${ }^{d}$ Durante flujos de aguas claras: Incremento máximo de $25 \mathrm{mg} / \mathrm{L}$ con respecto a la base para cualquier exposición corta (e.g. periodo de 24 horas). Incremento máximo promedio de $5 \mathrm{mg} / \mathrm{L}$ sobre los niveles base para periodos de exposición prolongados (mayores a 24 horas). Durante flujo altos o para aguas turbias: Incremento máximo de $25 \mathrm{mg} / \mathrm{L}$ con respecto a los niveles base cuando estos se encuentran entre 25 y $250 \mathrm{mg} / \mathrm{L}$. No deberá incrementarse más del $10 \%$ respecto a los niveles base, cuando la base $\geq 250 \mathrm{mg} / \mathrm{L}$.

e Total de $\mathrm{NH}^{3}(\operatorname{como} \mathrm{N})=1,216^{*}(0,019 /[1 / 10([(0,09101821+(2729,92 / \mathrm{T}))-\mathrm{pH}]+1)]) * 0,8224$

${ }^{f}$ Para flujos de aguas claras: Incremento máximo de 8 NTU sobre la base para periodos de exposición cortos (e.g. periodos de 24 horas). Incremento promedio máximo de 2 NTU a partir de los niveles base para exposiciones mayores a 24 horas. Para flujos turbulentos o de aguas turbias: Incremento máximo de 8 NTU sobre los niveles de base para cualquier tiempo en el que estos se encuentren entre 8 y 80 NTU. No deberá incrementarse más del 10\% por sobre los niveles de base cuando la base $>80$ NTU.

${ }^{8}$ Las adiciones térmicas no deberán alterar la estratificación térmica, exceder el promedio máximo semanal de temperatura, ni exceder la temperatura máxima a corto plazo.

'Incremento máximo de $20 \%$ en Unidades de Color (CU) por sobre sus condiciones naturales.

* Valor referencial al punto de control de inmisión en el cuerpo receptor 
concernientes al uso del agua dentro de las operaciones y el potencial de sus impactos ambientales deben tomarse con cautela.

- En tanto que, de otro lado, es necesario rescatar que aun cuando las condiciones necesarias para la implementación exitosa de la inyección de recortes en estratos subsuperficiales imposibilitan su aplicación cotidiana, la CRI continúa siendo la apuesta más segura para proyectos de perforación en áreas remotas o ambientalmente sensibles.

Respecto a la revisión de los límites permisibles en el manejo de descargas líquidas, se plantea las siguientes conclusiones:

- El trabajo comparativo, aquí presentado, entre la directriz ambiental ecuatoriana para las operaciones del sector de gas y petróleo, reportes gubernamentales de agencias ambientales y publicaciones científicas en materia de ecotoxicología en cuerpos acuáticos provee un terreno firme sobre el cual es posible afirmar que una gran parte del RAOHE aún se encuentra vigente en relación a los requerimientos de varios estándares internacionales sobre el manejo y disposición de descargas industriales líquidas.

- Las consideraciones sobre los límites respectivos a las descargas de efluentes líquidos y su respectivo empleo son una función de los procedimientos detallados en la política ambiental local, implicando que, si no existen los mecanismos de ejecución adecuados, la implementación de estos límites no concertará resultados.

\section{RECOMENDACIONES}

Para el fracturamiento hidráulico, primordialmente se recomienda evaluar a fondo el estado mecánico del pozo y la formación en cuestión, a fin de garantizar la apropiada contención y aislamiento de los fluidos inyectados y extraídos del estrato productor.

Para la reinyección de recortes, es relevante tomar en cuenta que aun cuando el devolver los ripios de perforación a su sitio de origen ha constituido un paso significativo en el cuidado del medio ambiente, la disposición o descarga de desechos no deja de ser el último eslabón en la gestión de residuos. Y por tanto, es relevante pensar prioritariamente en otros mecanismos que incluyan la minimización, reducción, reciclaje y recuperación, o tratamiento de los residuos.

En lo referente a la revisión de límites permisibles en el manejo de descargas líquidas, se recomienda mantener parámetros de control, con un número limitado de variables, que sean comprensibles para todas las partes involucradas en el control ambiental. Deberán evitarse parámetros que sean demasiado abstractos o sofisticados.

\section{REFERENCIAS}

Abou-Sayed, A., \& Guo, Q. (2001). Design Considerations in Drill Cuttings Re-Injection Through Downhole Fracturing. In SPE/IADC Middle East Drilling Technology Conference (p. 9). Bahrain: Society of Petroleum Engineers. https://doi.org/10.2118/72308-MS

Abou-Sayed, A., \& Guo, Q. (2002). Drilling and Production Waste Injection in Subsea Operations-Challenges and Recommendations. In Offshore Technology Conference 2002 (p. 8). Houston, TX: Society of Petroleum Engineers. https://doi.org/10.4043/14288-MS

Alabaster, J. S. (1982a). Dissolved Oxygen. In J. S. Alabaster (Ed.), Water Quality Criteria for Freshwater Fish (2nd ed., pp. 127-142). Stevenage: Butterworth-Heinemann. https://doi.org/10.1016/B978-0408-10849-2.50010-1

Alabaster, J. S. (1982b). Extreme pH value. In J. S. Alabaster (Ed.), Water Quality Criteria for Freshwater Fish (2nd ed., pp. 21-45). Stevenage: Butterworth-Heinemann. https://doi.org/10.1016/B978-0-408-108492.50006-X

Alberta Energy Regulator. (2018). Hydraulic Fracturing. Retrieved July 19, 2018, from https://www.aer.ca/providing-information/bytopic/hydraulic-fracturing

ALL Consulting, \& Ground Water Protection Council. (2009). Modern Shale Gas Development in the United Sates: A primer. Oklahoma City, OK.

American Petroleum Institute. (2009). HF1 - Hydraulic Fracturing Operations - Well Construction and Integrity Guidelines. Washington, DC. Retrieved from https://www.energyindepth.org/wpcontent/uploads/2009/03/API-HF.pdf

American Petroleum Institute. (2010). HF2 - Water Management Associated with Hydraulic Fracturing. Washington, DC: American Petroleum Institute.

American Petroleum Institute. (2013). Guidance/Best Practices Supporting Hydraulic Fracturing. American Petroleum Institute.

American Petroleum Institute. (2014). API Standards on Exploration and Production Operations.

Andagoya, K., \& Pérez, H. (2013). Estudio de la tecnología y el desarrollo para la explotación de gas metano de mantos carboníferos. [Tesis ingeniería]. Escuela Politécnica Nacional.

Barrios, P. (2012). CAPP's new guidelines for Canadian shale gas producers: A review of key requirements. Shareholder Association for Research \& Education.

Bruno, M., Reed, A., \& Olmstead, S. (2000). Environmental Management, Cost Management, and Asset Management for High-Volume Oil Field Waste Injection Projects. In IADC/SPE 2000 Drilling Conference (p. 10). New Orleans, Louisiana: Society of Petroleum Engineers.

Burgess, L. C. (2013). Organic Pollutants in Soil. In E. C. Brevik \& L. C. Burgess (Eds.), Soils and Human Health (2nd ed., pp. 83-106). Boca Raton, Florida: CRC Press.

Canadian Association of Petroleum Producers. (2012a). Baseline Groundwater Testing. CAPP Hydraulic Fracturing Operating Practice.

Canadian Association of Petroleum Producers. (2012b). Fracturing Fluid Additive Risk Assessment and Management. CAPP Hydraulic Fracturing Operating Practice.

Canadian Association of Petroleum Producers. (2012c). Water sourcing, measurement, and reuse. CAPP Hydraulic Fracturing Operating Practice.

Canadian Association of Petroleum Producers. (2017). Understanding Hydraulic fracturing. Canadian Association of Petroleum Producers.

Comisión OSPAR. (2001). Environmental aspects of on and off-site injection of drill cuttings and produced water. Londres.

Commision OSPAR. (2014). Establishment of a list of Predicted No Effect Concentrations (PNECS) for naturally occurring substances in produced water (OSPAR Agreement 2014-05).

Department of Environment and Heritage Protection. (2014). Barron River Basin Environmental Values and Water Quality Objectives. Queensland Government.

Department of Environment and Heritage Protection. (2014). Daintree and Mossman Rivers Basins Environmental Values and Water Quality Objectives. Queensland Government.

Doric, B., \& Dimovski, V. (2018). Managing petroleum sector performance a sustainable administrative design. Economic Research-Ekonomska Istraživanja, 31(1), 119-138. https://doi.org/10.1080/1331677X.2017.1421995

Environment and Sustainable Resource Development. (2014). Environment Quality Guidelines for Alberta Surface Waters. Canadá: AENVWeb.SWQ@gov.ab.ca. 
Environmental Performance Index. (2018). Global metrics for the environment: Ranking country performance on high-priority environmental issues, 1-4.

Frohlich, C., Potter, E., Hayward, C., \& Stump, B. (2010). Dallas-Ft Worth Earthquakes Coincident with Activity Associated with Natural Gas Production. The Leading Edge, 29(3), 270-275. https://doi.org/10.1190/1.3353720

Gallegos, T. J., Varela, B. A., Haines, S. S., \& Engle, M. A. (2015). Hydraulic fracturing water use variability in the United States and potential environmental implications. Water Resources Research, 51(7), 58395845. https://doi.org/10.1002/2015WR017278

Government of Canada. (2015). Wastewater Systems Effluent Regulations, Government of Canada § Canada.

Grant, S. B., Saphores, J.-D., Feldman, D. L., Hamilton, A. J., Fletcher, T. D., Cook, P. L. M., ... Marusic, I. (2012). Taking the "Waste" Out of "Wastewater" for Human Water Security and Ecosystem Sustainability. Science, 337(6095), 681-686. https://doi.org/10.1126/science.1216852

Gumarov, S., Shokanov, T., Simmons, S., Anokhin, V., \& Benelkadi, S. (2014). Good well design helps mitigate solids settling in cuttings reinjection - Drilling Contractor. Retrieved September 8, 2018, from http://www.drillingcontractor.org/good-well-design-helps-mitigatesolids-settling-in-cuttings-re-injection-30549

Guo, Q., \& Abou-Sayed, A. (2003). Worldwide Drill Cuttings Injection Permitting Requirements and Guidelines. In SPE/EPA/DOE Exploration and Production Environmental Conference (p. 8). San Antonio, TX: Society of Petroleum Engineers. https://doi.org/10.2118/80587-MS

Guo, Q., Geehan, T., \& Ovalle, A. (2007). Increased Assurance of Drill Cuttings Reinjection: Challenges, Recent Advances, and Case Studies. SPE Drilling \& Completion, 22(6), 99-105. https://doi.org/10.2118/87972-PA

Guo, Q., Geehan, T., \& Pincock, M. (2005). Managing Uncertainties and Risks in Drill-Cuttings Reinjection in Challenging Environments: Field Experience from Sakhalin. SPE/EPA/DOE Exploration and Production Environmental Conference. Galveston, Texas: Society of Petroleum Engineers. https://doi.org/10.2118/93781-MS

Harper, J. A. (2007). The Marcellus Shale - An Old "New" Gas Reservoir in Pennsylvania. Pennsylvania Geology, 38(1), 2-12.

Hernandez, R. M., Torres, M., Leal, J., Abad, F., Lopez, B., \& Bastidas, A (2015). Drill Cutting Reinjection Feasibility Study in a Critical Environment, Apaika Field, Ecuador. SPE Latin American and Caribbean Health, Safety, Environment and Sustainability Conference. Bogotá, Colombia: Society of Petroleum Engineers. https://doi.org/10.2118/174101-MS

Jarvie, H. P., Whitton, B. A., \& Neal, C. (1998). Nitrogen and phosphorus in east coast British rivers: Speciation, sources and biological significance. Science of The Total Environment, 210-211, 79-109. https://doi.org/10.1016/S0048-9697(98)00109-0

Jouanneau, S., Recoules, L., Durand, M. J., Boukabache, A., Picot, V., Primault, Y., ... Thouand, G. (2014). Methods for assessing biochemical oxygen demand (BOD): A review. Water Research, 49, 62-82. https://doi.org/10.1016/j.watres.2013.10.066

Keck, R. (2002). Drill Cuttings Injection: A Review of Major Operations and Technical Issues. In 2002 SPE Annual Technical Conference and Exhibition (p. 12). San Antonio, TX: Society of Petroleum Engineers. https://doi.org/10.2118/77553-MS

King, G. E. (2012). Hydraulic Fracturing 101: What Every Representative, Environmentalist, Regulator, Reporter, Investor, University Researcher, Neighbor and Engineer Should Know About Estimating Frac Risk and Improving Frac Performance in Unconventional Gas and Oil Wells. SPE Hydraulic Fracturing Technology Conference. The Woodlands, Texas, USA: Society of Petroleum Engineers. https://doi.org/10.2118/152596-MS

Kiparsky, M., \& Foley, J. (2013). Regulation of Hydraulic Fracturing in California: A Wastewater and Water Quality Perspective. Berkeley, CA.

Kondash, A., \& Vengosh, A. (2015). Water Footprint of Hydraulic Fracturing. Environmental Science \& Technology Letters, 2(10), 276-280. https://doi.org/10.1021/acs.estlett.5b00211

Lijzen, J. P. A., Baars, A. J., Otte, P. F., Rikken, M. G. J., Swartjes, F. A., Verbruggen, E. M. J., \& Van Wezel, A. P. (2001). Technical evaluation of the Intervention Values for Soil / Sediment and Groundwater. Bilthoven.
Liroff, R., Investor Environmental Health Network, Interfaith Center on Corporate, \& Responsibility. (2017). An Investor Guide to Disclosing Risks from Hydraulic Fracturing Operations.

Louisiana Department of Environmental Quality. (2017). Louisiana's Water Quality Regulations Part IX: Water Quality

Mauter, M. S., Alvarez, P. J. J., Burton, A., Cafaro, D. C., Chen, W., Gregory, K. B., ... Schnoor, J. L. (2014). Regional Variation in Water-Related Impacts of Shale Gas Development and Implications for Emerging International Plays. Environmental Science \& Technology, 48(15), 8298-8306. https://doi.org/10.1021/es405432k

Minister of Fisheries and Oceans. (2002). Metal Mining Effluent Regulations, Pub. L. No. SOR/2002-222

Ministerio de Ambiente y Desarrollo Sostenible. (2015). Resolución No. 0631. Bogotá, Colombia: MINAMBIENTE.

Ministerio de Energía y Minas. (2001). Reglamento Sustitutivo del Reglamento Ambiental para las Operaciones Hidrocarburíferas en el Ecuador. Ecuador.

Mordick, B. (2012). Hydraulic Fracturing Best Management Practices: Mitigating Environmental Concerns. In Hydraulic Fracturing $\mid$ A Wyoming Energy Forum (pp. 18-19). University of Wyoming.

Moschovidis, Z., Gardner, D. C., Sund, G. V, \& Veatch Jr., R. W. (1994). Disposal of Oily Cuttings by Downhole Periodic Fracturing Injections, Valhall, North Sea: Case Study and Modeling Concepts. SPE Drilling \& Completion, 9(04), 256-262. https://doi.org/10.2118/25757-PA

Nagel, N., \& Strachan, K. (1998). Implementation of Cuttings Reinjection at the Ekofisk Field. SPE/ISRM Rock Mechanics in Petroleum Engineering. Trondheim, Norway: Society of Petroleum Engineers. https://doi.org/10.2118/47218-MS

Palisch, T. T., Vincent, M., \& Handren, P. J. (2010). Slickwater Fracturing: Food for Thought. SPE Production \& Operations, 25(03), 327-344. https://doi.org/10.2118/115766-PA

Romero, L., Ramirez, O., Hernandez, R., \& Bastidas, A. (2016). First Successful Cuttings Reinjection CRI Operation in Ecuador: An Alternative to Help Minimize the Environmental Impact in a Protected Area. In Abu Dhabi International Petroleum Exhibition \& Conference (p. 8). Abu Dhabi, EAU: Society of Petroleum Engineers. https://doi.org/10.2118/183150-MS

Rutqvist, J., Birkholzer, J., Cappa, F., \& Tsang, C. (2007). Estimating maximum sustainable injection pressure during geological sequestration of $\mathrm{CO} 2$ using coupled fluid flow and geomechanical fault- slip analysis. Energy Conversion and Management, 48(6), 17981807. https://doi.org/10.1016/j.enconman.2007.01.021

Saasen, A., Tran, T. N., Jøranson, H., Meyer, E., Gabrielsen, G., \& Tronstad, A. E. (2001). Subsea Re-Injection of Drilled Cuttings - Operational Experience. In SPE/IADC Drilling Conference (p. 6). Amsterdam, Países Bajos: Society of Petroleum Engineers. https://doi.org/10.2118/67733-MS

Safari, G., Yetilmezsoy, K., Mahvi, A., \& Zarrabi, M. (2013). Post-treatment of secondary wastewater treatment plant effluent using a two-stage fluidized bed bioreactor system. Journal of Environmental Health Science and Engineering, 11(1), 10. https://doi.org/10.1186/2052336X-11-10

Satterfield, J., Mantell, M., Kathol, D., Hiebert, F., Patterson, K., \& Lee, R. (2008). Managing Water Resource's Challenges in Select Natural Gas Shale Plays. In GWPC Annual Meeting (p. 8). Ground Water Protection Council.

Sparling, D. W. (2016a). Basics of Toxicity Testing. In D. W. B. T.-E. E. Sparling (Ed.), Ecotoxicology Essentials (pp. 21-44). San Diego: Academic Press. https://doi.org/10.1016/B978-0-12-801947-4.000020

Sparling, D. W. (2016b). Metals. In D. W. B. T.-E. E. Sparling (Ed.) Ecotoxicology Essentials (pp. 225-275). San Diego: Academic Press. https://doi.org/10.1016/B978-0-12-801947-4.00008-1

The Royal Society, \& Royal Academy of Engineering. (2012). Shale gas extraction in the UK: a review of hydraulic fracturing. Londres.

US Agency for Toxic Substances and Disease Registry. (2007). Toxicological Profile for Lead. US Department of Health and Human Services (Vol. 1). Atlanta, Georgia.

U.S. Environmental Protection Agency. (2004). Evaluation of Impacts to Underground Sources of Drinking Water by Hydraulic Fracturing of Coalbed Methane Reservoirs.

U.S. Environmental Protection Agency. (2018). Toxic and Priority Pollutants Under the Clean Water Act. Retrieved July 30, 2018, from https://www.epa.gov/eg/toxic-and-priority-pollutants-under-cleanwater-act\#priority 
U.S. Geological Survey. (2014). Hydraulic Fracturing (Fracking) FAQs. Retrieved from https://web.archive.org/web/20141019024105/http://www.usgs.gov/f $\mathrm{aq} / \mathrm{q}=$ categories $\% 2 \mathrm{~F} 10132 \% 2 \mathrm{~F} 3830$

Vengosh, A., Jackson, R. B., Warner, N., Darrah, T. H., \& Kondash, A. (2014). A critical review of the risks to water resources from unconventional shale gas development and hydraulic fracturing in the United States. Environmental Science \& Technology, 48(15), 83348348. https://doi.org/10.1021/es405118y

Viswanathan, H. S., Carey, J. W., Karra, S., Porter, M. L., Rougier, E., Currier, R. P., ... Hyman, J. D. (2015). Integrated Experimental and Computational Study of Hydraulic Fracturing and the Use of Alternative Fracking Fluids. 49th U.S. Rock Mechanics/Geomechanics Symposium. San Francisco, California: American Rock Mechanics Association.

Voutsa, D., Manoli, E., Samara, C., Sofoniou, M., \& Stratis, I. (2001). A Study of Surface Water Quality in Macedonia, Greece: Speciation of Nitrogen and Phosphorus. Water, Air, and Soil Pollution, 129(1), 1332. https://doi.org/10.1023/A:1010315608905

Weston, R. T. (2008). Development of the Marcellus Shale-Water Resource Challenges.

World Health Organization. (2004). Guidelines for Drinking-Water Quality (3rd ed.). Ginebra: SNPBT Typesetter.

WorleyParsons. (2014). Environmental Regulation. An International Comparison of Leading Oil and Gas Producing Regions. Canadian Association of Petroleum Producers.

Zehner, R. (2012). Reglamento Sustitutivo del Reglamento Ambiental para las Operaciones Hidrocarburíferas en el Ecuador: Introducción, Enfoques y Objetivos. Quito: Ministerio de Energía y Minas.

\section{BIOGRAFÍAS}

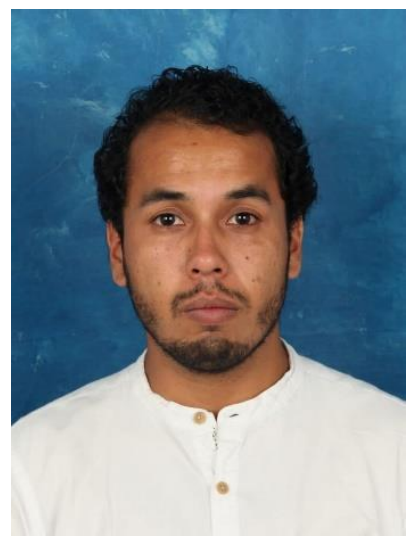

Daniel Sebastián Castillo Castro, Ingeniero en Petróleos de la Escuela Politécnica Nacional. Actualmente, se encuentra cursando sus estudios de Máster en Ingeniería Ambiental en la National Cheng Kung University, Taiwán. Sus áreas de investigación incluyen: desarrollo sostenible, análisis de ciclo de vida, análisis de ciclo de vida y teledetección (remote sensing).

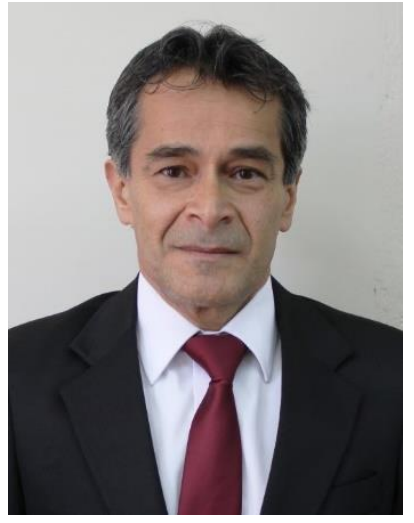

Johnny Robinson Zambrano Carranza, Profesor adscrito al Departamento de Petróleos, Escuela Politécnica Nacional. Las áreas de investigación son en Ingeniería Ambiental, Termoquímica y Química Física. Actualmente trabaja en métodos para la producción y recuperación de crudo pesado.

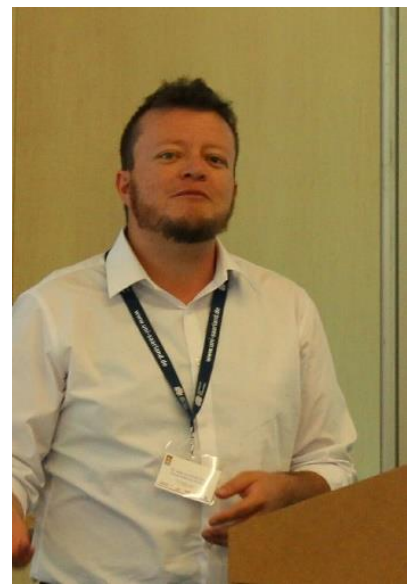

José Luis Rivera Parra, Biólogo de la PUCE y con un doctorado en Ecología y Evolución de la Universidad de Missouri en Saint Louis. Interesado en ecología de comunidades y en la mitigación de impactos ambientales causados por industrias extractivas en ecosistemas tropicales. 
Revista Politécnica, Noviembre 2021 - Enero 2022, Vol. 48, No. 2 